\title{
Highly aligned, large pore ordered mesoporous carbon films by solvent vapor annealing with soft shear
}

Zhe Qiang, Yuanzhong Zhang, Yi Wang, Sarang M. Bhaway, Kevin A. Cavicchi ${ }^{*}$ and Bryan D. Vogt*

Department of Polymer Engineering, University of Akron, Akron, OH 44325

Mailing address for all authors:

Department of Polymer Engineering, University of Akron, Polymer Engineering Academic Center, 250 S. Forge St., Akron, OH 44325

\begin{abstract}
Macroscopic alignment of block copolymer (BCP)-templated mesoporous carbon films is challenging, especially for large pores $(>10 \mathrm{~nm})$, due to their slow dynamics that impede re-orientation of the ordered domains. Here, we demonstrate a facile method, solvent vapor annealing with soft shear (SVA-SS), to fabricate unidirectionally aligned, ordered mesoporous carbon films using two different $\mathrm{BCP}$ templates, poly(ethylene oxide)-block-poly(n-butyl acrylate) and polystyrene-blockpoly(styrenesulfonate $\mathrm{N}, \mathrm{N}$-dimethyl- $n$-octadecylamine), and both cylindrical and spherical morphologies with relatively large accessible pores $(\approx 15 \mathrm{~nm})$. This alignment is preserved through the thermopolymerization of resol and carbonization. The alignment of the mesopores impacts several key properties of these carbon films, especially for the unidirectional cylindrical mesostructures. The highly aligned mesoporous carbon films exhibit a more narrow pore size distribution than the analogous unaligned ordered

${ }^{*}$ Corresponding author: Email: vogt@uakron.edu (B. D. Vogt), kac58@ uakron.edu (K.A. Cavicchi). Tele: 330.972 .8608 (B.D. Vogt), 330.972 .8368 (K.A. Cavicchi)
\end{abstract}


mesoporous carbon as determined by ellipsometric porosimetry. Moreover, the electrical conductivity of the films becomes anisotropic with nearly $40 \%$ difference in conductivity between parallel and perpendicular to the orientation direction of the cylindrical mesopores. In the parallel orientation, the electrical conductivity is over $20 \%$ greater than the analogous unoriented (random) films. These results illustrate the applicability of SVA-SS to obtain unidirectional aligned mesoporous carbon films over large areas without additional physical or chemical templating.

\section{INTRODUCTION}

Ordered mesoporous carbon (OMC) materials have received significant interest due to their unique combination of properties, including high surface area [1]; high electrical conductivity [2]; chemical inertness; and easily tunable pore sizes [3, 4]. These materials are attractive for a broad range of applications, such as supercapacitors [5], fuel cells [6], absorbents [7], heterogeneous catalyst supports [8], and drug delivery [9, 10]. Ordered mesoporous carbon is generally fabricated through one of two templated synthesis routes: hard-templating based on the replication of an inorganic master (typically silica) [11, 12] or soft-templating based on the cooperative assembly of surfactants or block copolymers with carbon precursors [13-16]. These soft-templated, ordered mesoporous carbons have been synthesized in a variety of forms as monoliths [17, 18], particles [19, 20], fibers [21], powders [22, 23], thin films [24-26], and free-standing membranes [27]. However, changes in the geometry and form of these templated materials can require alteration in the fabrication protocols in order to achieve the same ordered structure. For example, the compositional windows for well defined, single ordered morphologies is altered in thin 
films in comparison to bulk powders [25]. Additionally, pioneering work by Bein and coworkers demonstrated that ordering occurs during thermopolymerization of resol for films [28], instead of evaporation induced self-assembly [29, 30]. This dynamic nature of the mesostructure in films provides opportunities for improved control of the ordered structure, but the thermal energy that enables ordering of the nanostructure also drives the crosslinking of the resol precursor. This leads to a competition between the crosslinking and ordering kinetics that can limit the quality of the ordered structures [31].

The initial report of soft-templated ordered mesoporous carbon used solvent vapor annealing (SVA) to produce the highly ordered mesostructure, which likely drove orientation of the cylindrical mesopores through the thickness of the film [32]. We have recently illustrated that the kinetic frustrations with ordering from the cooperative assembly of resol with a high molecular block copolymer template can be overcome by an analogous SVA process $[31,33]$. However, these self-assembled mesostructures lack registration over macroscopic dimensions with randomly oriented ordered grains. This relatively short-ranged ordered structure can adversely impact the performance of mesoporous carbons in electrochemical applications [34, 35], so development of techniques to align the ordered structure may provide routes to further improve performance.

Several techniques have been proposed and demonstrated for the long-range alignment of ordered mesoporous carbon films. Matyjaszewski and coworkers developed long range orientation of a perpendicular lamellae morphology using zone-casting on a hot stage to 
make line space patterns of carbon, but this method has been only demonstrated for a single poly(acrylonitrile)-containing block copolymer [36]. Cold zone annealing (CZA) has been demonstrated to enable fabrication of unidirectional aligned cylindrical and spherical mesostructured carbon films (FDU-15 and FDU-16, respectively) [37] and the preferential in-plane orientation can be tuned by the processing parameters [38]. However, the efficacy of alignment of the mesostructure decreases significantly as the mobility of the polymer mixture (both templates and resol) decreases [37], which limits its applicability for aligning the mesoporous carbon films with relatively large pore size (>10 nm). There have been a number of reports for large pore, ordered mesoporous carbons fabricated by cooperative assembly with a block copolymer [39-42], but their long-range alignment in thin films has not been demonstrated. In order to address these shortcomings, it would be useful to work within the constraints that what ever method is utilized that it should be generalizable to multiple block copolymer systems that are cooperatively assembled with resol, easy to implement, and finally, do not require the use of specialized facilities or substrates. As these prior alignment techniques for mesoporous carbons as well as some for mesoporous silicate [43] films rely on techniques originally developed for neat BCP films [44-47], it is useful to consider some alternative techniques that have been previously developed for BCPs.

To address the limitations associated with the alignment of large pore ordered mesoporous carbon films, we apply the solvent vapor annealing with soft shear method (SVA-SS), which has been recently demonstrated as a facile method for BCP alignment $[48,49]$. By simply placing a soft elastomer slab in contact with the film surface during 
SVA, the solvent vapor then swells both the templated film to provide mobility for rearrangement of the domains and the elastomer to provide a directional force for the alignment upon solvent removal. Poly(ethylene oxide)-block-poly(n-butyl acrylate) (PEO- $b$-PBA) and polystyrene-block-poly(styrenesulfonate N,N-dimethyl- $n$ octadecylamine) (PS- $b$-PSS-DOMDA), are examined to address the broad applicability of SVA-SS to align the soft-templated mesoporous carbon with relatively large pore size (> $10 \mathrm{~nm}$ ) based on the cooperative assembly with resol for both cylindrical and spherical nanostructures from each template. This alignment of the mesostructure impacts critical properties of the mesoporous carbon films. The aligned cylindrical mesoporous carbon thin films exhibit a sharper pore size distribution than the unaligned analog and exhibit anisotropic electrical conductivity with a $20 \%$ increase in conductivity parallel to the alignment direction when compared to analogous mesoporous carbon film without alignment.

\section{Experimental Section}

\subsection{Materials}

Tetrahydrofuran (THF) (> $99 \%$ ), phenol (> $99 \%$ ), formaldehyde (ACS reagent, $37 \mathrm{wt} \%$ in $\mathrm{H}_{2} \mathrm{O}$, contains $10-15 \%$ methanol as stabilizer), and 2,2-azobis(2-methylpropionitrile) (AIBN) were purchased from Sigma-Aldrich. $n$-Butyl acrylate (BA, $>99.5 \%$, stabilized) was purchased from Acros. Methyl-ethyl-ketone (MEK) (> $99 \%)$ and hexane (> $99 \%)$ were purchased from Fisher Scientific. Sodium hydroxide (> 99\%) was purchased from EMD Chemicals Inc. Styrene (>99\%, stabilized with 10-15 ppm 4-tert- butylcatechol) 
was purchased from Alfa Aesar. Basic alumina was purchased from Sigma Aldrich. Nitrogen gas (> $99 \%$ ) was purchased from Praxair and used in both the polymer synthesis and carbonization. Styrene and BA monomers were purified by passing through through basic $\mathrm{Al}_{2} \mathrm{O}_{3}$ columns to remove the inhibitor. AIBN was recrystallized by dissolving in methanol at $45{ }^{\circ} \mathrm{C}$ and recrystallized in a freezer. A poly(ethylene oxide) macro-reversible addition fragmentation chain transfer (RAFT) agent (PEO-RAFT) was synthesized as described in a previous report [50]. All other reagents were used as received.

PEO- $b$-PBA was synthesized using RAFT polymerization (see Supplementary Material), the mole fraction of PBA in the PEO- $b$-PBA is $75 \mathrm{~mol} \%$ and the molecular weight determined by gel permeation chromatography (GPC) is $M_{n}=26.4 \mathrm{~kg} / \mathrm{mol}$ based on polystyrene (PS) standards. PS- $b$-PSS-DMODA $\left(\mathrm{M}_{\mathrm{n}}=40.2 \mathrm{~kg} / \mathrm{mol}, \mathrm{f}_{\mathrm{PS}}=0.52, \mathrm{PDI}=\right.$ 1.28) was synthesized by RAFT polymerization as previously reported [51]. Phenolformaldehyde resin (resol) was synthesized under alkaline condition following the standard protocol described for organic-organic self-assembly [13], but dissolved in THF instead of ethanol for the stock solution.

Poly(dimethyl siloxane), PDMS, (Sylgard 184, Dow Corning) was prepared at 10:1 w/w ratio of base to curing agent with the components physically mixed by hand. The mixture was poured onto a flat glass plate. The PDMS was allowed to cure and degas at room temperature for $1 \mathrm{~h}$, followed by curing at $120^{\circ} \mathrm{C}$ for $2 \mathrm{~h}$. The cured PDMS was cut into rectangular slabs $(3 \mathrm{~cm} \times 1 \mathrm{~cm})$ to act as soft capping layers for the polymer films. 


\subsection{Films fabrication and processing}

Mesoporous films were prepared by organic-organic self-assembly of PS- $b$-PSSDMODA and resol (precursor) at a mass ratio of 1:1 for cylindrical mesostructures and 1:2 for spherical mesostructures. Similarly for the PEO- $b$-PBA template, the mass ratios were 1:2 and 1:3.5 for cylindrical and spherical mesostructures, respectively. For fabricating the films, silicon wafers with a $300 \mathrm{~nm}$ thick thermal oxide (University Wafer) were used as substrates. The wafers were cleaned using piranha solution $(7: 3 \mathrm{v} / \mathrm{v}$ of $\mathrm{H}_{2} \mathrm{SO}_{4}: 30 \% \mathrm{H}_{2} \mathrm{O}_{2}$ ) at $90{ }^{\circ} \mathrm{C}$ for 50 min, followed by rinsing with deionized water 3 times. Thin films were spun cast from $10 \mathrm{wt} \%$ (solids) THF solution at $3500 \mathrm{rpm}$ for 1 $\min$.

Post film fabrication processing employed solvent vapor annealing with soft shear (SVASS) [49]. PDMS slabs were adhered to the surface of the spun cast films and then the polymer film-PDMS assembly was exposed to saturated solvent vapor for $2 \mathrm{~h}$ using mass flow controllers (MKS-146C-FF000-1) at $800 \mathrm{~mL} / \mathrm{min}$. Subsequently, the assembly was dried using air flowing at $25 \mathrm{~mL} / \mathrm{min}$ for $30 \mathrm{~min}$. Then the PDMS slabs were removed from BCP-resol films. Two solvents were utilized for this annealing process: MEK for PS- $b$-PSS-DMODA:resol films and THF for PEO- $b$-PBA:resol films. Following SVA-SS, the films were heated at $150{ }^{\circ} \mathrm{C}$ for $24 \mathrm{~h}$ to crosslink the resol and the crosslinked films were then carbonized at $800{ }^{\circ} \mathrm{C}$ (heating schedule of $1{ }^{\circ} \mathrm{C} / \mathrm{min}$ to $600{ }^{\circ} \mathrm{C}, 4{ }^{\circ} \mathrm{C} / \mathrm{min}$ for $>$ $600{ }^{\circ} \mathrm{C}$, and held at $800{ }^{\circ} \mathrm{C}$ for $3 \mathrm{~h}$ ) under $\mathrm{N}_{2}$ atmosphere. 
Control experiments were performed using SVA without the PDMS to provide mobility without an alignment force. Similarly, the advantage of the SVA-SS process for alignment of large pore materials was demonstrated by comparison to cold zone annealing (CZA), which has been demonstrated to be effective for alignment of mesoporous carbon films using commercial templates (Pluronics®) [37]. The details for the CZA process employed here were $2 \mu \mathrm{m} / \mathrm{s}$ and maximum temperature of $120{ }^{\circ} \mathrm{C}$ (determined by infrared camera, Testo 875).

\subsection{Characterization}

The optical constants $(n, k)$ and thickness of the films were elucidated using variable angle spectroscopic ellipsometry (VASE, J.A. Woollam Co., M-2000) with the wavelength ranging from $246 \mathrm{~nm}$ to $1689 \mathrm{~nm}$. The ellipsometric angles ( $\Delta$ and $\Psi$ ) were fit using a recursive model consisting of the silicon substrate, an interface layer, $\mathrm{SiO}_{2}$ layer for the oxide and a Cauchy layer to describe the polymeric films. For the carbonized films, the large adsorption in the visible regime was fit with a generalized oscillator model consisting of Gaussian and Lorenzian functions. The surface morphology of the films after each processing step was investigated by atomic force microscopy (AFM, Dimension ICON, Veeco). Micrographs were obtained in tapping mode at $0.5 \mathrm{~Hz}$ using PPP-NCC-50 tips (Nanosensors). Defect density of spherical nanostructures was determined using Voronoi diagrams. For the Voronoi diagrams presented herein, the blue dots are used to signify spheres without 6 nearest neighbors that are expected for the BCC structure. 
Grazing incidence small angle X-ray scattering (GISAXS) measurements were performed at X9 beamline of National Synchrotron Light Source (NSLS) at Brookhaven National Laboratory (BNL). An incident X-ray beam of energy $13.5 \mathrm{keV}(\lambda=0.0918 \mathrm{~nm})$ was used. Samples were measured under vacuum $(\approx 40 \mathrm{~Pa})$. All of films were measured below and above critical angle with data collected using a Pilatus detector at a distance of $5 \mathrm{~m}$. Rotational GISAXS was performed by rotating the sample stage in a range of $180^{\circ}$ in order to provide a full scan of mesostructure orientation. The in-plane primary diffraction peak was utilized to determine $I(\phi)$ for the rotational GISAXS in order to assess the Hermans orientation factor [52]. Ellipsometric porosimetry (EP) was used to determine the porosity and pore size distribution (PSD) of the PEO-b-PBA templated mesoporous carbon films. For EP measurements, toluene was used as the probe solvent; the mass flow controllers (MKS-146C-FF000-1) were used to control its vapor pressure by mixing the saturated solvent vapor with a dry air stream. The change in refractive index as a function of relative pressure is converted to relative volume fraction of toluene absorbed in the mesoporous films using Lorentz-Lorenz effective medium approximation (EMA)

$$
\frac{V_{a b}}{V_{f i l m}}=\frac{\left(\frac{n^{2}-1}{n^{2}+2}\right)-\left(\frac{n_{0}^{2}-1}{n_{0}^{2}+2}\right)}{\left(\frac{n_{t o l}{ }^{2}-1}{n_{t o l}{ }^{2}+2}\right)}
$$

where $n_{0}$ and $n_{\text {tol }}$ is the refractive index (at $632 \mathrm{~nm}$ ) of empty mesoporous films and toluene $\left(n_{\mathrm{tol}}=1.496\right)$ at respectively. The pore size distribution for cylindrical pores was estimated from adsorption curve using the Kelvin equation,

$$
\frac{2}{r_{k}}=\frac{1}{r_{1}}+\frac{1}{r_{2}}=-\frac{R T}{\gamma V_{L} \cos \theta} \ln \left(\frac{P}{P 0}\right)
$$


where $r_{1}$ and $r_{2}$ are the radius of curvature of meniscus, $r_{k}$ is the Kelvin radius, $R$ is the gas constant, $T$ is the temperature, $\gamma$ is the surface tension of toluene, $V_{L}$ is the molar volume of the toluene, and $\theta$ is the contact angle of toluene and assuming the physical

properties of toluene are unchanged from the bulk, $\gamma$ is $0.0284 \mathrm{~N} \mathrm{~m}^{-1} ; V_{L}$ is $1.064 \times 10^{-4} \mathrm{~m}^{3}$ $\mathrm{mol}^{-1}$; and contact angle is $\approx 0^{\circ} . \mathrm{X}$-ray photoelectron spectroscopy (XPS, PHI5000 Versa Probe II Scanning XPS Microprobe, ULVAC-PHI, Inc.) and Fourier transform infrared spectroscopy (Thermo Scientific iS50 FT-IR) performed to determine the composite of the carbonized films. The conductivity of mesoporous carbon films along different direction was determined by a four-point probe system (Lucas Lab PR04-4400). The spacing between each probe is 0.04 inch with the current controlled by the outer probes that induce a potential between the inner probes that is measured.

\section{Results and discussion}

Figure 1 schematically illustrates the SVA-SS process that is used to align the selfassembled nanostructures. In this process, a rectangular PDMS slab is first adhered to the film. By introducing the solvent vapor, the PDMS and films are both simultaneously swollen. The plasticization of the BCP templates and resol by the sorbed solvent enhances its mobility for ordering. Subsequently, a dry air stream establishes a unidirectional drying front from the corner of PDMS slabs that leads to the development of well-defined shear. This shear force induces alignment of the BCP domains along the diagonal direction of the PDMS pad and the direction for the aligned BCP domains can be effectively controlled by simply changing the shape and placement of the PDMS slab [49]. 


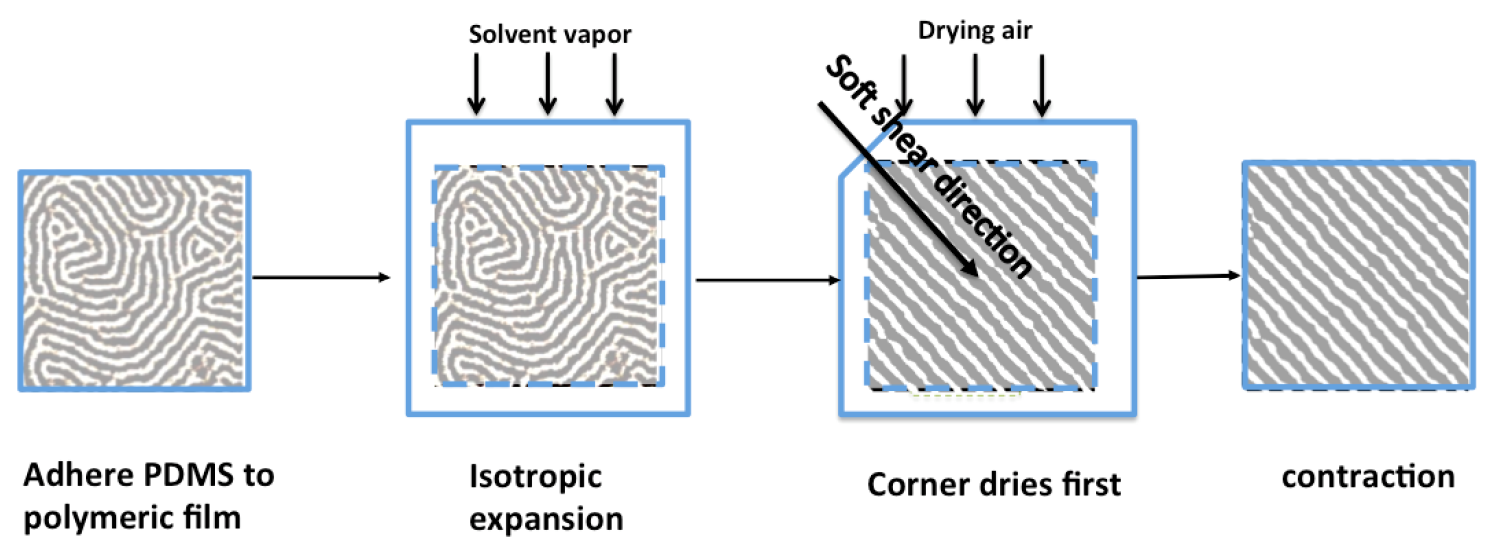

Figure 1. Schematic of the swelling and deswelling process during the SVA-SS for polymer films alignment.

Figure 2A illustrates the surface morphology of the as-cast PEO- $b$-PBA:resol films at 1:2 mass ratio. This morphology is short ranged ordered parallel cylinders with the characteristic fingerprint patterns, consistent with the diffused isotropic FFT ring in the inset of Figure 2A. Alternatively after SVA-SS, the surface morphology of the PEO-b-PBA:resol films consists of a highly correlated stripe patterns that persist across the films as shown in Figure 2B. The FFT of the micrograph illustrates the orientation by two orders of diffraction spots. For the PEO- $b$-PBA: resol examined here, CZA does not lead to any preferential alignment in the ordered mesostructure of the film (see Supplementary Material), which is attributed to the limited chain mobility of the relatively high molecular weight of PEO- $b$-PBA templates $\left(\mathrm{M}_{\mathrm{n}}=25,000 \mathrm{~g} / \mathrm{mol}\right)$. This is consistent with previous studies of CZA where the orientation factor significantly decreases when the molecular weight of BCP templates increases [37]. Standard SVA leads to an increase in the long-range order of the mesostructure, but there is no preferential 
alignment of these features (see Supplementary Material). These mesostructures developed during SVA-SS are maintained after thermopolymerization at $150{ }^{\circ} \mathrm{C}$ for $24 \mathrm{~h}$ (see Supplementary Material) and carbonization at $800{ }^{\circ} \mathrm{C}$ in $\mathrm{N}_{2}$. Figure $2 \mathrm{C}$ shows the AFM phase images of SVA-SS aligned films after carbonization, the cylinder-to-cylinder spacing on the surface shrinks from $29 \mathrm{~nm}$ (after thermopolymerization) to $24 \mathrm{~nm}$, indicating approximately $15 \%$ in-plane shrinkage during the carbonization process and the resultant pore width of the cylinders determined from the surface morphology is approximately $12 \mathrm{~nm}$. The backside of these films can be examined after transfer of the films from the substrate to reveal an analogous stripe pattern. This indicates both the free surface and substrate surface are highly aligned by SVA-SS (see Supplementary Material). By increasing the resol loading to produce a hydrophilic fraction of $85 \%$, spherical mesostructures can be fabricated. Figure 2D shows the SVA-SS aligned spherical forming nanostructures templated by PEO- $b$-PBA after carbonization. It should be noted that the perfection in the orientation is significantly less for the spherical morphology in comparison to the cylinders, which is not completely unexpected based on the block copolymer literature for the shear alignment of BCC mesostructures associated with thickness above 2 layers [53]. Despite this shortcoming in the alignment of the spherical mesostructure, there is a significant decrease in the defect density of resultant mesoporous carbon films from $45 \mu \mathrm{m}^{-2}$ (without SVA-SS) to $7 \mu \mathrm{m}^{-2}$ (with SVA-SS) [54]. 

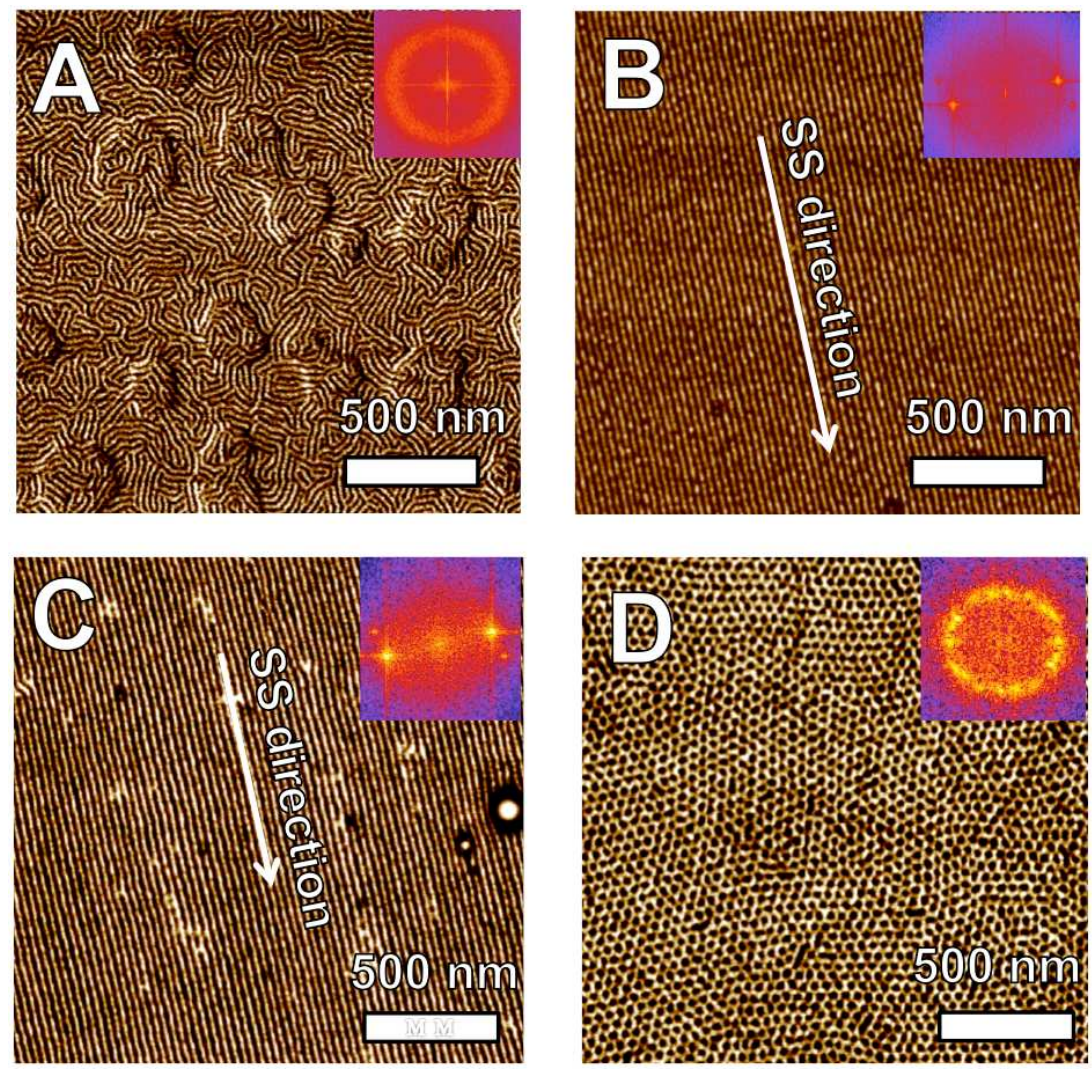

Figure 2. AFM phase images of cylindrical forming PEO-b-PBA:resol for (A) as cast , (B) after SVA-SS films and (C) after carbonization. A highly ordered mesostructure is also obtained for (D) spherical forming PEO-b-PBA: resol film after SVA-SS and carbonization. THF was utilized as the solvent in both cases. The insets in the micrographs illustrate the FFT of the AFM image.

While the AFM micrographs pictorially illustrate the local surface structure and alignment, the alignment over more macroscopic dimensions can be better determined using rotational GISAXS [55]. For the quantification of the orientation, the direction of alignment (diagonal direction of PDMS slab) is defined as $\phi=0^{\circ}$. When the incident beam is parallel to the alignment $\left(\phi=0^{\circ}\right)$, the GISAXS pattern (Figure 3A) shows multiple diffraction peaks that indicates the highly ordered structure and enable the 
unambiguous determination of the space group: hexagonal packing $(\mathrm{p} 6 \mathrm{~mm})$ of cylindrical domains. However, when the sample is rotated to the $\phi=90^{\circ}$ (Figure 3B), only one weak primary peak can be ascertained from the scattering. By plotting the normalized scattering intensity of primary order peak of mesoporous carbon films as a function of azimuthal angle for cylinders (Figure 3C), only one single peak can be observed indicating their preferred orientation and the Herman's orientation factor [52] determined from GISAXS for cylinders is $S=0.88$.
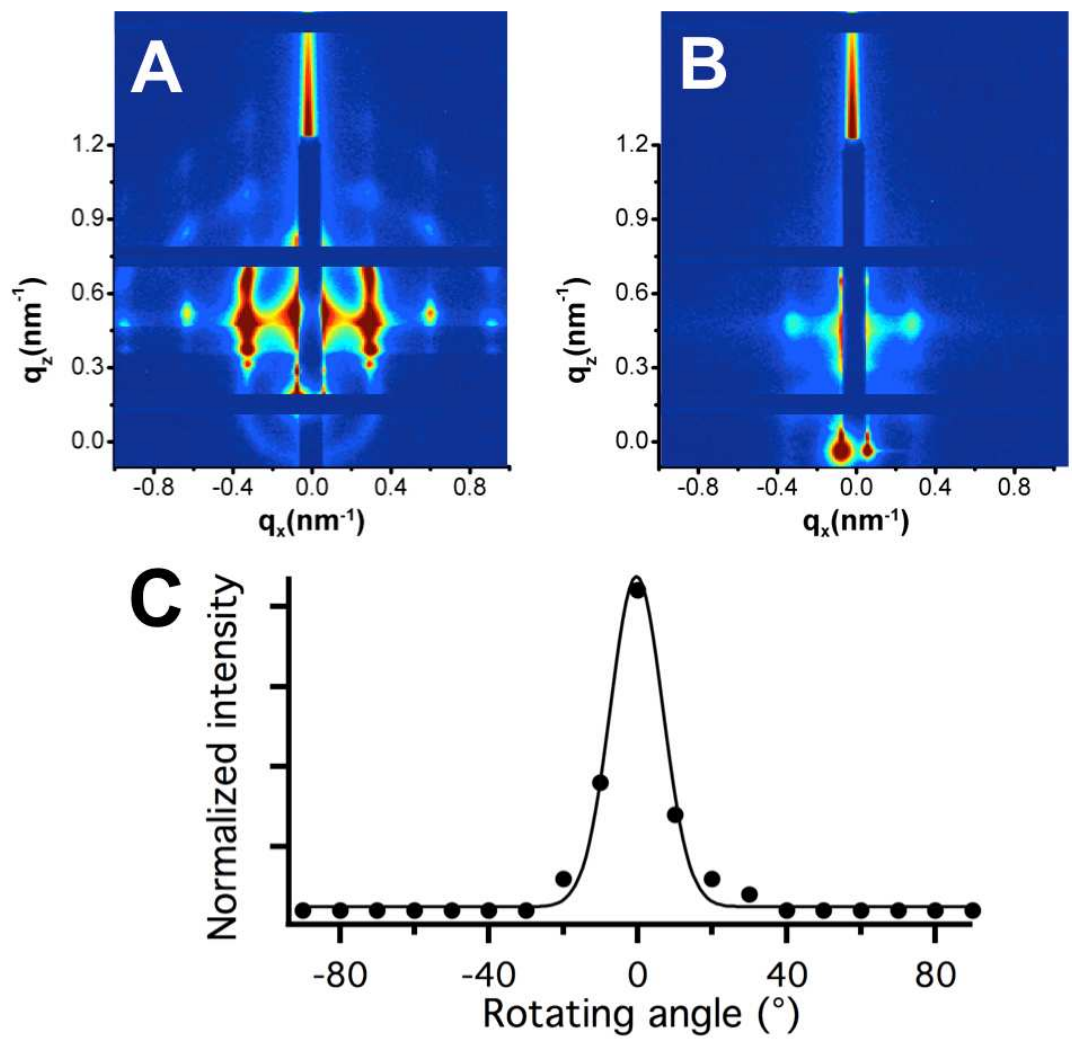

Figure 3. GISAXS profiles for cylindrical forming PEO- $b$-PBA templated mesoporous carbon film for (A) $\phi=0^{\circ}$ and (B) $\phi=90^{\circ}$; (C) shows the normalized primary peak intensity as a function of azimuthal angle. 
To illustrate the broad applicability of SVA-SS to align the soft-templated mesoporous carbon, SVA-SS with an ionic block copolymer template, PS- $b$-PSS-DMODA, is demonstrated with very limited ordering in the as-cast PS- $b$-PSS-DMODA:resol films (see Supplementary Material) as reported previously [51]. In this case, by applying the SVA-SS method, a cylindrical mesoporous carbon film shows highly aligned stripe patterns, which can be clearly observed in the AFM micrographs (Figure 4A). For the spherical nanostructures (Figure 4B), the defect density of spheres after SVA-SS $\left(9 \mu \mathrm{m}^{-2}\right)$ decreases significantly from the standard SVA process $\left(21 \mu \mathrm{m}^{-2}\right)$ as determined by Voronoi analysis. Thus, this method provides a route to orient the mesostructure in ordered mesoporous carbon films, but it is unclear how these changes would impact the properties of the material.

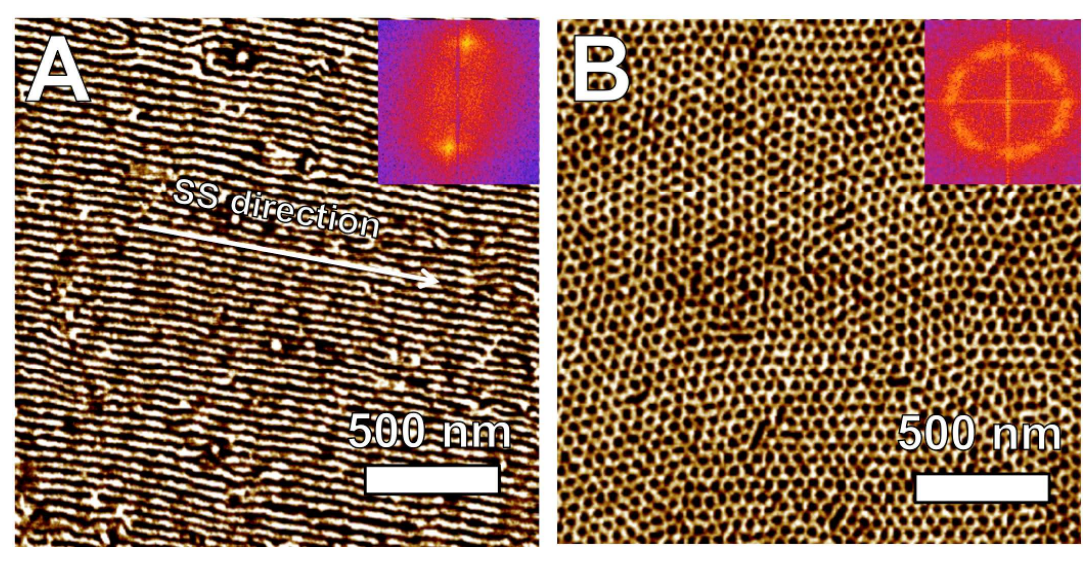

Figure 4. AFM phase images of SVA-SS aligned PS- $b$-PSS-DMODA:resol films (A) cylindrical forming and (B) spherical forming after carbonization. MEK was utilized as the solvent in both cases.

First, the alignment process should not impact the composition of the carbon obtained. Figure 5A shows the XPS survey spectra of SVA-SS aligned PEO- $b$-PBA templated 
mesoporous carbon thin film; there are three peaks associated with $\mathrm{O} 1 \mathrm{~s}, \mathrm{C} 1 \mathrm{~s}$ and $\mathrm{Si} 2 \mathrm{~s}$ (from substrate), which confirms no residual metal in the film from PDMS slab. The carbon is predominately $\mathrm{sp}^{2}$ from the peak at $284 \mathrm{eV}$. Interestingly, the XPS spectrum of PS- $b$-PSS-DMODA (Figure 5B) templated mesoporous carbon shows a very small peak associated with nitrogen besides the expected carbon and oxygen as well as the silicon substrate. A small peak at $286 \mathrm{eV}$ confirms this $\mathrm{C}-\mathrm{N}$ bond. We attribute this slight nitrogen doping to the PSS-DMODA segment of the template. This nitrogen functionality has the potential to enhance the electrical performance of the mesporous carbon due to its electron-donor properties and electrode wettability [56, 57]. The XPS measurement also provides the elemental composition of mesoporous carbon films. The $\mathrm{C}: \mathrm{O}$ ratio in both cases is approximately 1:0.1, which is slightly higher than reported previously for FDU15 processed at $800{ }^{\circ} \mathrm{C}$. [13] Additionally, the FT-IR spectra of the mesoporous carbon films templated by PEO- $b$-PBA are identical for standard SVA treatment and SVA-SS treatment is identical. This is consistent with no residual containment from SVA-SS process. (see Supplementary Material) 

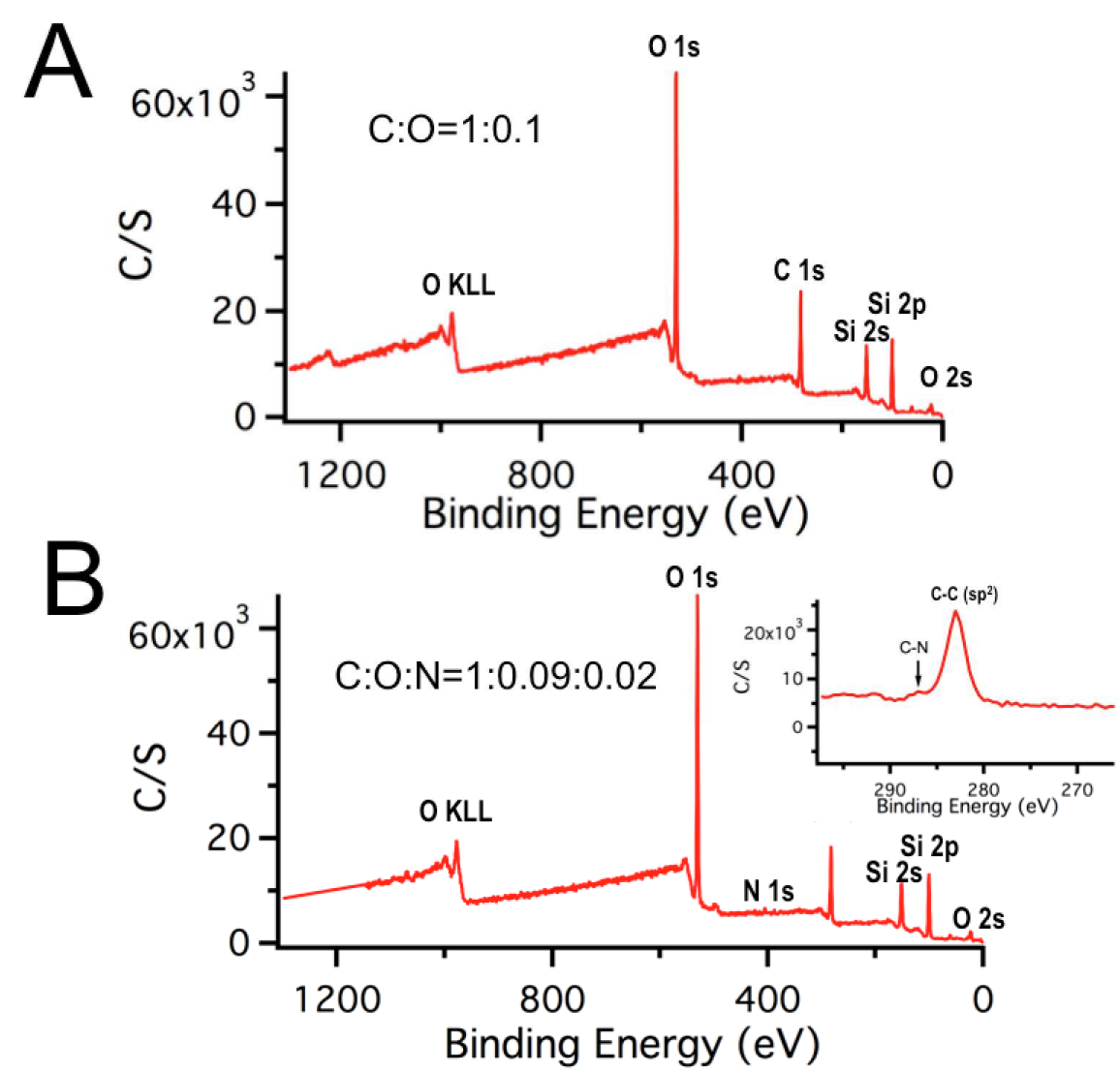

Figure 5. XPS spectra of mesoporous carbon thin films templated by (A) PEO- $b$-PBA and (b) PS- $b$-PSS-DMODA.

To investigate how SVA-SS impacts the porosity and the pore size distribution of the mesoporous carbon films, PEO- $b$-PBA templated cylindrical mesoporous carbon films are examined using ellipsometric porosimetry (EP). The porosity of non-SVA film is approximately $23 \%$ and the porosity increases to $27 \%$ when SVA is applied. This increase is attributed to improvements in to the ordered structure that decrease the film contraction during the carbonization, which is consistent with the previous results [31]. However, there is no significant difference in the porosity between the mesoporous carbon films annealed by SVA and SVA-SS (28\%). However, the curvature of the sorption $\left(\mathrm{V}_{\mathrm{ab}} / \mathrm{V}_{\mathrm{film}}\right)$ isotherms is more strongly dependent on the processing details. For the non-SVA films 
(Figure $6 \mathrm{~A}$ ), $\mathrm{V}_{\mathrm{ab}} / \mathrm{V}_{\text {film }}$ increases slowly during adsorption as vapor pressure increases with no plateau in the sorption capacity. During desorption, the mesopores empty over a broad range of partial pressures. These two features suggest at least a modest breadth to the distribution of pore sizes. Conversely, the SVA treated (random oriented) film shows a sharp increase in $\mathrm{V}_{\mathrm{ab}} / \mathrm{V}_{\text {film }}$ at $\mathrm{p} / \mathrm{p}_{0} \approx 0.7$, which corresponds to capillary condensation of the toluene probe in mesopores of a well-defined size. Highly aligned mesoporous carbon (SVA-SS film) exhibits a sharper hysteresis loop in the desorption isotherm compared to the both non-SVA film and SVA-film, which is consistent with prior observations on correlations between the sharpness of the hysteresis and the extent of ordering [58]. Additionally, for both SVA film and SVA-SS film, the $\mathrm{V}_{\mathrm{ab}} / \mathrm{V}_{\text {film }}$ plateaus at high vapor pressure indicating no large macropores. The derivative of the $\mathrm{V}_{\mathrm{ab}} / \mathrm{V}_{\text {film }}$ with respect to $r_{k}$ provides an estimate of the pore size distribution based on the Kelvin equation as shown in Figure 6B. Without SVA, a broad pore size distribution is observed with a small peak at approximately $r_{k}=7.8 \mathrm{~nm}$. The peak sharpens with SVA, which indicates more uniform pore sizes due to the improvement of ordering in the films. Additionally, the peak shifts to larger pore size $\left(r_{k}=10 \mathrm{~nm}\right)$. This shift is likely due to the swelling of the polymer chains during SVA [31]. The average pore size does not change with SVA-SS when compared to the standard SVA; however, the pore population becomes tighter, which illustrates the improvement in the uniformity of the pore size with alignment. 

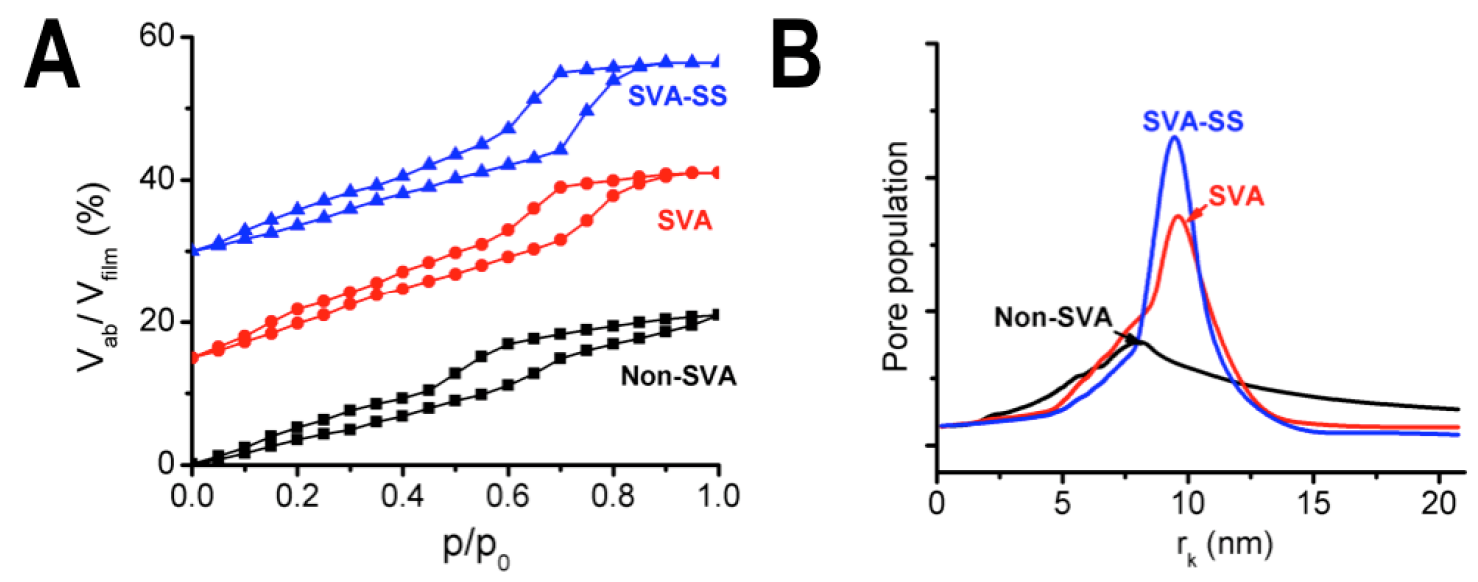

Figure 6. (A) Adsorption and desorption isotherm of PEO- $b$-PBA templated mesoporous carbon films under different processing conditions. SVA and SVA-SS are vertically offset by $15 \%$ and $30 \%$. (B) The pore size distribution from these adsoption isotherms as derived from Kelvin equation.

To investigate whether the alignment of nanostructure leads to anisotropic properties, the conductivity of SVA-SS aligned cylindrical mesoporous carbon (PEO- $b$-PBA templated) is carefully examined as the electrodes are rotated from $0^{\circ}$ (current flow parallel to the alignment direction) to $90^{\circ}$ (current flow perpendicular to the alignment direction). As the matrix is carbon in all cases, directional dependence of the conductivity might not be expected, but conductivity of the aligned samples is dependent on the electrode orientation as shown in Figure 7. The isotropic mesoporous carbon (SVA only) exhibits electrical conductivity of approximately $18.5 \mathrm{~S} / \mathrm{cm}$, which is consistent with previous reports of FDU-16 carbonization at $800{ }^{\circ} \mathrm{C}$ [59]. For the highly aligned cylindrical mesoporous carbon thin film, when the current flow is parallel to the alignment direction, the sample shows the highest conductivity that is approximately $23 \mathrm{~S} / \mathrm{cm}$. The conductivity slightly decreases monotonically as the sample is rotated away from parallel 
alignment and the conductivity reaches the minimum value $(15 \mathrm{~S} / \mathrm{cm})$ when the current flow is perpendicular to the alignment direction. Although the increase in conductivity with alignment of mesoporous carbon $(21 \%)$ compared to that for the isotropic orientation is small, this slight anisotropy might prove useful for some applications [60].

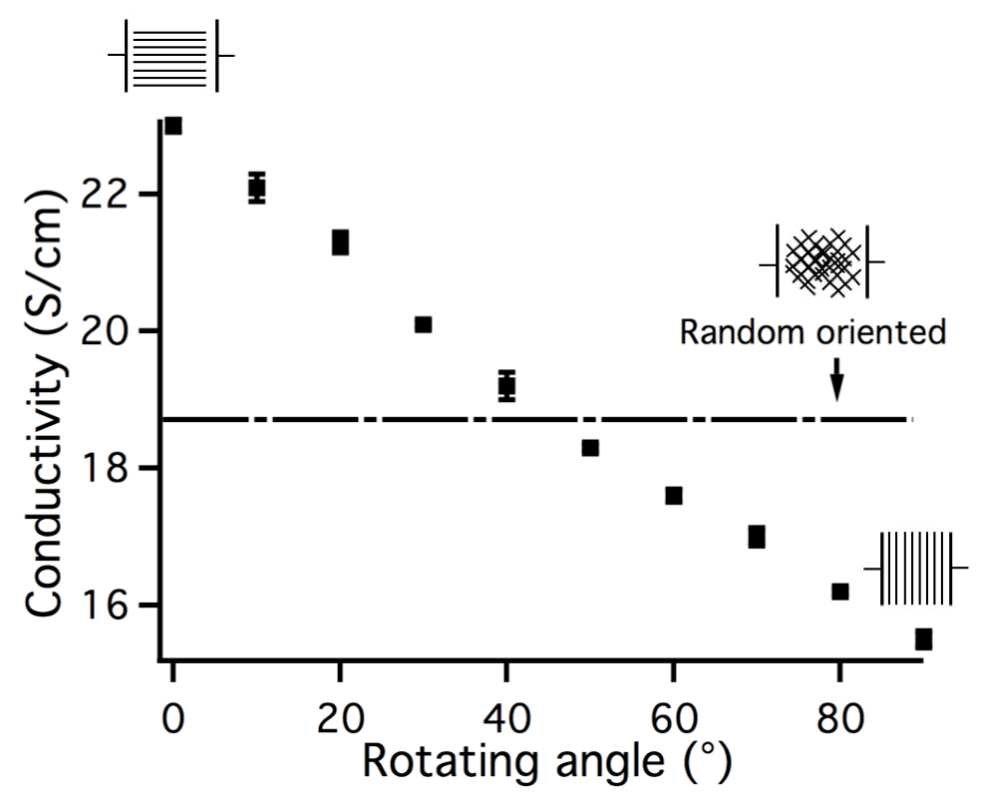

Figure 7. Conductivity of aligned meosporous carbon films as a function of the orientation of the electrodes to the alignment direction of the cylindrical mesopores.

\section{Conclusions}

Solvent vapor annealing with soft shear has been applied to synthesize highly aligned mesoporous carbon thin film with both cylindrical and spherical morphologies by using two different templates: PEO- $b$-PBA and PS- $b$-PSS-DMODA. In both cases, the alignment is preserved during carbonization. The alignment of mesoporous carbon films results in a more narrow pore size distribution and anisotropic electrical conductivity. The 
conductivity of cylindrical mesoporous carbon thin film can be improved over $20 \%$ by aligning the cylinders using SVA-SS method. These results indicate the SVA-SS is an effective approach for obtaining unidirectional aligned mesoporous carbon thin films over large areas with different pore size.

\section{Acknowledgements}

This work is partial supported by the National Science Foundation under grant CBET1336057. The authors thank Kevin Yager for his assistance with the GISAXS measurements. Use of the National Synchrotron Light Source, Brookhaven National Laboratory, was supported by the U.S. Department of Energy, Office of Science; Office of Basic Energy Sciences, under Contract No. DE-AC02-98CH10886. Research carried out at the Center for Functional Nanomaterials, Brookhaven National Laboratory, is supported by the U.S. Department of Energy, Office of Basic Energy Sciences, under Contract No. DE-AC02-98CH10886.

\section{References}

[1] Liu RL, Shi YF, Wan Y, Meng Y, Zhang FQ, Gu D, et al. Triconstituent Coassembly to ordered mesostructured polymer-silica and carbon-silica nanocomposites and large-pore mesoporous carbons with high surface areas. J. Am. Chem. Soc. 2006;128(35):11652-11662. 
[2] Zhai YP, Dou YQ, Zhao DY, Fulvio PF, Mayes RT, Dai S. Carbon Materials for Chemical Capacitive Energy Storage. Adv. Mater. 2011;23(42):4828-4850.

[3] Labiano A, Dai MZ, Young WS, Stein GE, Cavicchi KA, Epps TH, et al. Impact of Homopolymer Pore Expander on the Morphology of Mesoporous Carbon Films Using Organic-Organic Self-Assembly. J. Phys. Chem. C. 2012;116(10):6038-6046.

[4] Deng YH, Yu T, Wan Y, Shi YF, Meng Y, Gu D, et al. Ordered mesoporous silicas and carbons with large accessible pores templated from amphiphilic diblock copolymer poly(ethylene oxide)-b-polystyrene. J. Am. Chem. Soc. 2007;129(6):1690-1697.

[5] Feng D, Lv YY, Wu ZX, Dou YQ, Han L, Sun ZK, et al. Free-Standing Mesoporous Carbon Thin Films with Highly Ordered Pore Architectures for Nanodevices. J. Am. Chem. Soc. 2011;133(38):15148-15156.

[6] Chang H, Joo SH, Pak C. Synthesis and characterization of mesoporous carbon for fuel cell applications. J. Mater. Chem. 2007;17(30):3078-3088.

[7] Levario TJ, Dai MZ, Yuan W, Vogt BD, Nielsen DR. Rapid adsorption of alcohol biofuels by high surface area mesoporous carbons. Microporous Mesoporous Mater. 2012;148(1):107-114.

[8] Yu JS, Kang S, Yoon SB, Chai G. Fabrication of ordered uniform porous carbon networks and their application to a catalyst supporter. J. Am. Chem. Soc. 2002;124(32):9382-9383.

[9] Wang SB. Ordered mesoporous materials for drug delivery. Microporous Mesoporous Mater. 2009;117(1-2):1-9.

[10] Saha D, Warren KE, Naskar AK. Soft-templated mesoporous carbons as potential materials for oral drug delivery. Carbon. 2014;71:47-57. 
[11] Ryoo R, Joo SH, Jun S. Synthesis of highly ordered carbon molecular sieves via template-mediated structural transformation. J. Phys. Chem. B. 1999;103(37):7743-7746. [12] Jun S, Joo SH, Ryoo R, Kruk M, Jaroniec M, Liu Z, et al. Synthesis of new, nanoporous carbon with hexagonally ordered mesostructure. J. Am. Chem. Soc. 2000;122(43):10712-10713.

[13] Meng Y, Gu D, Zhang FQ, Shi YF, Cheng L, Feng D, et al. A family of highly ordered mesoporous polymer resin and carbon structures from organic-organic selfassembly. Chem. Mater. 2006;18(18):4447-4464.

[14] Deng YH, Liu C, Yu T, Liu F, Zhang FQ, Wan Y, et al. Facile synthesis of hierarchically porous carbons from dual colloidal crystal/block copolymer template approach. Chem. Mater. 2007;19(13):3271-3277.

[15] Ryoo R, Joo SH, Kruk M, Jaroniec M. Ordered mesoporous carbons. Adv. Mater. 2001;13(9):677-681.

[16] Tanaka S, Nishiyama N, Egashira Y, Ueyama K. Synthesis of ordered mesoporous carbons with channel structure from an organic-organic nanocomposite. Chem. Comm. 2005(16):2125-2127.

[17] Hao GP, Li WC, Wang SA, Wang GH, Qi L, Lu AH. Lysine-assisted rapid synthesis of crack-free hierarchical carbon monoliths with a hexagonal array of mesopores. Carbon. 2011;49(12):3762-3772.

[18] Liang CD, Dai S. Dual Phase Separation for Synthesis of Bimodal Meso/Macroporous Carbon Monoliths. Chem. Mater. 2009;21(10):2115-2124. 
[19] Schuster J, He G, Mandlmeier B, Yim T, Lee KT, Bein T, et al. Spherical Ordered Mesoporous Carbon Nanoparticles with High Porosity for Lithium-Sulfur Batteries. Angew. Chem. Int Ed. 2012;51(15):3591-3595.

[20] Liu J, Yang TY, Wang DW, Lu GQM, Zhao DY, Qiao SZ. A facile soft-template synthesis of mesoporous polymeric and carbonaceous nanospheres. Nature Comm. 2013;4. doi:10.1038/ncomms3798

[21] Tanaka S, Doi A, Nakatani N, Katayama Y, Miyake Y. Synthesis of ordered mesoporous carbon films, powders, and fibers by direct triblock-copolymer-templating method using an ethanol/water system. Carbon. 2009;47(11):2688-2698.

[22] Teng W, Wu Z, Fan J, Chen H, Feng D, Lv Y, et al. Ordered mesoporous carbons and their corresponding column for highly efficient removal of microcystin-LR. Energ Environ Sci. 2013;6(9):2765-2776.

[23] Liang CD, Dai S. Synthesis of mesoporous carbon materials via enhanced hydrogen-bonding interaction. J. Am. Chem. Soc. 2006;128(16):5316-5317.

[24] Tanaka S, Nakatani N, Doi A, Miyake Y. Preparation of ordered mesoporous carbon membranes by a soft-templating method. Carbon. 2011;49(10):3184-3189.

[25] Song L, Feng D, Fredin NJ, Yager KG, Jones RL, Wu Q, et al. Challenges in Fabrication of Mesoporous Carbon Films with Ordered Cylindrical Pores via Phenolic Oligomer Self-Assembly with Triblock Copolymers. ACS Nano. 2010;4(1):189-198.

[26] Tanaka S, Katayama Y, Tate MP, Hillhouse HW, Miyake Y. Fabrication of continuous mesoporous carbon films with face-centered orthorhombic symmetry through a soft templating pathway. J Mat Chem. 2007;17(34):3639-3645. 
[27] Feng D, Lv Y, Wu Z, Dou Y, Han L, Sun Z, et al. Free-Standing Mesoporous Carbon Thin Films with Highly Ordered Pore Architectures for Nanodevices. J. Am. Chem. Soc. 2011;133(38):15148-15156.

[28] Schuster J, Kohn R, Doblinger M, Keilbach A, Amenitsch H, Bein T. In Situ SAXS Study on a New Mechanism for Mesostructure Formation of Ordered Mesoporous Carbons: Thermally Induced Self-Assembly. J. Am. Chem. Soc. 2012;134(27):1113611145.

[29] Kawashima D, Aihara T, Kobayashi Y, Kyotani T, Tomita A. Preparation of mesoporous carbon from organic polymer/silica nanocomposite. Chem. Mater. 2000;12(11):3397-3401.

[30] Brinker CJ, Dunphy DR. Morphological control of surfactant-templated metal oxide films. Current Opinion in Colloid \& Interface Science. 2006;11(2-3):126-132.

[31] Qiang Z, Xue JC, Cavicchi KA, Vogt BD. Morphology Control in Mesoporous Carbon Films Using Solvent Vapor Annealing. Langmuir. 2013;29(10):3428-3438.

[32] Liang CD, Hong KL, Guiochon GA, Mays JW, Dai S. Synthesis of a large-scale highly ordered porous carbon film by self-assembly of block copolymers. Angew. Chem. Int. Ed. 2004;43(43):5785-5789.

[33] Deng G, Zhang Y, Ye C, Qiang Z, Stein GE, Cavicchi K, et al. Bicontinuous mesoporous carbon thin films via an order-order transition. Chem Commun. 2014. DOI: $\underline{10.1039 / \mathrm{C} 4 \mathrm{CC} 02471 \mathrm{~K}}$

[34] Matsui T, Tanaka S, Miyake Y. Correlation between the capacitor performance and pore structure of ordered mesoporous carbons. Adv. Powder Technol. $2013 ; 24(4): 737-742$. 
[35] Tanaka S, Doi A, Matsui T, Miyake Y. Mass transport and electrolyte accessibility through hexagonally ordered channels of self-assembled mesoporous carbons. J. Power Sources. 2013;228:24-31.

[36] Tang CB, Tracz A, Kruk M, Zhang R, Smilgies DM, Matyjaszewski K, et al. Long-range ordered thin films of block copolymers prepared by zone-casting and their thermal conversion into ordered nanostructured carbon. J. Am. Chem. Soc. 2005;127(19):6918-6919.

[37] Xue J, Singh G, Qiang Z, Karim A, Vogt BD. Unidirectional self-assembly of soft templated mesoporous carbons by zone annealing. Nanoscale. 2013;5(17):7928-7935.

[38] Xue JC, Singh G, Qiang Z, Yager KG, Karim A, Vogt BD. Facile control of long range orientation in mesoporous carbon films with thermal zone annealing velocity. Nanoscale. 2013;5(24):12440-12447.

[39] Wickramaratne NP, Jaroniec M. Phenolic resin-based carbons with ultra-large mesopores prepared in the presence of poly(ethylene oxide)-poly(butylene oxide)poly(ethylene oxide) triblock copolymer and trimethyl benzene. Carbon. 2013;51:45-51.

[40] Shim J, Lee J, Ye Y, Hwang J, Kim SK, Lim TH, et al. One-Pot Synthesis of Intermetallic Electrocatalysts in Ordered, Large-Pore Mesoporous Carbon/Silica toward Formic Acid Oxidation. ACS Nano. 2012;6(8):6870-6881.

[41] Deng Y, Cai Y, Sun Z, Gu D, Wei J, Li W, et al. Controlled Synthesis and Functionalization of Ordered Large-Pore Mesoporous Carbons. Adv. Funct. Mater. 2010;20(21):3658-3665.

[42] Zhang JY, Deng YH, Wei J, Sun ZK, Gu D, Bongard H, et al. Design of Amphiphilic ABC Triblock Copolymer for Templating Synthesis of Large-Pore Ordered 
Mesoporous Carbons with Tunable Pore Wall Thickness. Chem. Mater. 2009;21(17):3996-4005.

[43] Koganti VR, Dunphy D, Gowrishankar V, McGehee MD, Li X, Wang J, et al. Generalized coating route to silica and titania films with orthogonally tilted cylindrical nanopore arrays. Nano Lett. 2006;6(11):2567-2570.

[44] Morkved TL, Lu M, Urbas AM, Ehrichs EE, Jaeger HM, Mansky P, et al. Local control of microdomain orientation in diblock copolymer thin films with electric fields. Science. 1996;273(5277):931-933.

[45] Singh G, Yager KG, Smilgies D-M, Kulkarni MM, Bucknall DG, Karim A. Tuning Molecular Relaxation for Vertical Orientation in Cylindrical Block Copolymer Films via Sharp Dynamic Zone Annealing. Macromolecules. 2012;45(17):7107-7117.

[46] Tang C, Wu W, Smilgies D-M, Matyjaszewski K, Kowalewski T. Robust Control of Microdomain Orientation in Thin Films of Block Copolymers by Zone Casting. J. Am. Chem. Soc. 2011;133(30):11802-11809.

[47] Davis RL, Chaikin PM, Register RA. Cylinder Orientation and Shear Alignment in Thin Films of Polystyrene-Poly(n-hexyl methacrylate) Diblock Copolymers. Macromolecules. 2014;47(15):5277-5285.

[48] Qiang Z, Zhang YZ; Groff J; Cavicchi KA; Vogt BD. A generalized method for alignment of block copolymer films: Solvent vapor annealing with soft shear. Soft Matter. 2014. doi:10.1039/C4SM00875H

[49] Qiang Z, Zhang LH; Cavicchi KA; Vogt BD. Unidirectional Alignment of Block Copolymer Films Induced by Expansion of a Permeable Elastomer during Solvent Vapor Annealing. Macromolecules. 2014;47(3):1109-1116. 
[50] Deng G, Qiang Z, Lecorchick W, Cavicchi KA, Vogt BD. Nanoporous Nonwoven Fibril-Like Morphology by Cooperative Self-Assembly of Poly(ethylene oxide)-blockPoly(ethyl acrylate)-block-Polystyrene and Phenolic Resin. Langmuir. 2014;30(9):25302540.

[51] Qiang Z, Xue J, Cavicchi KA, Vogt BD. Morphology control in mesoporous carbon films using solvent vapor annealing. Langmuir 2013;29(10):3428-3438.

[49] Angelescu DE, Waller JH, Register RA, Chaikin PM. Shear-induced alignment in thin films of spherical nanodomains. Adv. Mater. 2005;17(15):1878-1881.

[50] Segalman RA. Patterning with block copolymer thin films. Mater. Sci. Eng., R 2005;48(6):191-226.

[51] Qiang Z, Xue J, Stein GE, Cavicchi KA, Vogt BD. Control of Ordering and Structure in Soft Templated Mesoporous Carbon Films by Use of Selective Solvent Additives. Langmuir. 2013;29(27):8703-8712.

[52] J. J. Hermans PHH, D. Vermaas, A. Weidinger. Quantitative evaluation of orientation in cellulose fibres from the X-ray fibre diagram. Recueil des Travaux Chimiques des Pays-Bas. 1946;65(6):427-447.

[53] Angelescu DE, Waller JH, Register RA, Chaikin PM. Shear-induced alignment in thin films of spherical nanodomains. Adv Mater. 2005;17(15):1878-1881.

[54] Segalman RA. Patterning with block copolymer thin films. Mater Sci \& Eng RReports. 2005;48(6):191-226.

[55] Salzmann I, Moser A, Oehzelt M, Breuer T, Feng X, Juang Z-Y, et al. Epitaxial Growth of pi-Stacked Perfluoropentacene on Graphene-Coated Quartz. ACS Nano. 2012;6(12):10874-10883. 
[56] Kim ND, Kim W, Joo JB, Oh S, Kim P, Kim Y, et al. Electrochemical capacitor performance of $\mathrm{N}$-doped mesoporous carbons prepared by ammoxidation. J Power Sources. 2008;180(1):671-675.

[57] Kichambare P, Kumar J, Rodrigues S, Kumar B. Electrochemical performance of highly mesoporous nitrogen doped carbon cathode in lithium-oxygen batteries. J Power Sources. 2011;196(6):3310-3316.

[58] Li X, Song L, Vogt BD. Tuning mechanical properties of mesoporous silicas using associating homopolymers/block copolymer blends as templates. J Phys Chem C. 2008;112(1):53-60.

[59] Li X, Larson AB, Jiang L, Song L, Prichard T, Chawla N, et al. Evolution of mechanical, optical and electrical properties of self-assembled mesostructured phenolic resins during carbonization. Microporous and Mesoporous Mater. 2011;138(1-3):86-93.

[60] Zhi J, Zhao W, Liu X, Chen A, Liu Z, Huang F. Highly Conductive Ordered Mesoporous Carbon Based Electrodes Decorated by 3D Graphene and 1D Silver Nanowire for Flexible Supercapacitor. Adv Funct Mater. 2014;24(14):2013-2019. 